\title{
Quantitative analysis of transformed ray transferences of optical systems in a space of augmented Hamiltonian matrices*
}

\section{WF Harris}

Optometric Science Research Group, Department of Optometry, University of Johannesburg, PO Box 524, Auckland Park, 2006 South Africa

<wfh@uj.ac.za>

\begin{abstract}
There is a need for methods for quantitative analysis of the first-order optical character of optical systems including the eye and components of the eye. Because of their symplectic nature ray transferences themselves are not closed under addition and multiplication by a scalar and, hence, are not amenable to conventional quantitative analysis such as the calculation of an arithmetic mean. However transferences can be transformed into augmented Hamiltonian matrices which are amenable to such analysis. This paper provides a general methodology and in particular shows how to calculate means and variance-covariances representing the first-order optical character of optical systems. The systems may be astigmatic and may have decentred elements. An accompanying paper shows application to the cornea of the human eye with allowance for thickness.
\end{abstract}

The ray transference $\mathbf{T}$, a $5 \times 5$ matrix in the most general case of astigmatic and decentred systems, can be transformed into an augmented Hamiltonian matrix in a variety of ways. ${ }^{1-3}$ Because augmented Hamiltonian matrices of the same order constitute a linear or vector space we are provided with a natural machinery for standard quantitative analysis of the first-order optical character of optical systems. The purpose of this paper is to show how. An accompanying paper ${ }^{4}$ applies the methods described here to a particularly simple system, the cornea of the human eye.

In transformed form the transference $\mathbf{T}$ can be written as

$$
\hat{\mathbf{T}}=\left(\begin{array}{ccc}
\hat{\mathbf{A}} & \hat{\mathbf{B}} & \hat{\mathbf{e}} \\
\hat{\mathbf{C}} & \hat{\mathbf{D}} & \hat{\pi} \\
\mathbf{o}^{\prime} & \mathbf{o}^{\prime} & \tilde{0}
\end{array}\right) .
$$

The submatrix

$$
\hat{\mathbf{S}}=\left(\begin{array}{ll}
\hat{\mathbf{A}} & \hat{\mathbf{B}} \\
\hat{\mathbf{C}} & \hat{\mathbf{D}}
\end{array}\right)
$$

is Hamiltonian, that is, it satisfies

$$
\hat{\mathbf{S}}^{\prime} \mathbf{E}=\mathbf{E}^{\prime} \hat{\mathbf{S}}
$$

where

$$
\mathbf{E}=\left(\begin{array}{cc}
\mathbf{O} & \mathbf{I} \\
-\mathbf{I} & \mathbf{O}
\end{array}\right),
$$

*Paper originally presented at Mopane 2006A, Visual Optics, an international conference held at Mopani Camp, Kruger National Park, Limpopo Province, South Africa, August 6-9 2006.

Received 18 October 2006; revised version accepted 12 June 2007 
$\mathbf{E}^{\prime}$ is the transpose of $\mathbf{E}$ and $\mathbf{O}$ and $\mathbf{I}$ are the $2 \times 2$ null and identity matrices respectively. The transformed transference $\hat{\mathbf{T}}$ is called an augmented Hamiltonian matrix ${ }^{3}$ because it consists of a Hamiltonian submatrix $\hat{\mathbf{S}}$ augmented by a fifth row of 0 s and a fifth column containing $\hat{\mathbf{e}}$ and $\hat{\pi}$. Equation 1 imposes restrictions on the submatrices $\hat{\mathbf{A}}, \hat{\mathbf{B}}, \hat{\mathbf{C}}$ and $\hat{\mathbf{D}}$ of $\hat{\mathbf{S}}$, namely that

$$
\hat{\mathbf{D}}=-\hat{\mathbf{A}}^{\prime}
$$

and that $\hat{\mathbf{B}}$ and $\hat{\mathbf{C}}$ are both symmetric.

One can readily show that the set of transformed transferences $\hat{\mathbf{T}}$ satisfies the axioms of a linear or vector space over the field of real numbers, the operations of the space being conventional matrix addition and multiplication of a matrix by a scalar. Of the 25 entries in $\hat{\mathbf{T}}$ the five in the bottom row are trivial. Of the remaining 20 entries only 14 are independent: equation 3 accounts for a reduction by four and the symmetric nature of $\hat{\mathbf{B}}$ and $\hat{\mathbf{C}}$ each by another one. Thus the space of transformed transferences is 14dimensional.

The space naturally identifies five subspaces. The first contains the transformed transferences of conjugate afocal systems. It includes all transformed transferences of the form

$$
\left(\begin{array}{ccc}
\hat{\mathbf{A}} & \mathbf{O} & \mathbf{0} \\
\mathbf{O} & -\hat{\mathbf{A}}^{\prime} & \mathbf{0} \\
\mathbf{o}^{\prime} & \mathbf{o}^{\prime} & \tilde{0}
\end{array}\right) .
$$

The subspace is evidently four dimensional. The second (three dimensional) contains all transformed transferences of the form

$$
\left(\begin{array}{ccc}
\mathbf{O} & \hat{\mathbf{B}} & \mathbf{0} \\
\mathbf{O} & \mathbf{O} & \mathbf{0} \\
\mathbf{o}^{\prime} & \mathbf{o}^{\prime} & 0
\end{array}\right)
$$

and includes those of homogeneous gaps. The third (also three dimensional) has the form

$$
\left(\begin{array}{ccc}
\mathbf{O} & \mathbf{O} & \mathbf{0} \\
\hat{\mathbf{C}} & \mathbf{O} & \mathbf{0} \\
\mathbf{o}^{\prime} & \mathbf{o}^{\prime} & 0
\end{array}\right)
$$

and includes the transformed transferences of all centred (that is, non-decentred) thin systems. The fourth (two dimensional) is the subspace of the form

$$
\left(\begin{array}{ccc}
\mathbf{O} & \mathbf{O} & \hat{\mathbf{e}} \\
\mathbf{O} & \mathbf{O} & \mathbf{0} \\
\mathbf{0}^{\prime} & \mathbf{o}^{\prime} & 0
\end{array}\right) \text {. }
$$

Such systems cause a transverse displacement of rays. Finally there is the subspace (also two dimensional) that contains all thin prisms and flat tilted surfaces; the transformed transferences have the form

$$
\begin{aligned}
& \left(\begin{array}{lll}
\mathbf{O} & \mathbf{O} & \mathbf{0} \\
\mathbf{O} & \mathbf{O} & \hat{\pi} \\
\mathbf{o}^{\prime} & \mathbf{o}^{\prime} & 0
\end{array}\right) . \\
& \hat{\mathbf{A}}=\left(\begin{array}{ll}
\hat{a}_{11} & \hat{a}_{12} \\
\hat{a}_{21} & \hat{a}_{22}
\end{array}\right) \text { expands naturally as } \\
& \hat{\mathbf{A}}=\hat{A}_{\mathrm{I}} \mathbf{I}+\hat{A}_{\mathrm{J}} \mathbf{J}+\hat{A}_{\mathrm{K}} \mathbf{K}+\hat{A}_{\mathrm{L}} \mathbf{L}
\end{aligned}
$$


where $\mathbf{I}$ is defined above, $\mathbf{J}=\left(\begin{array}{cc}1 & 0 \\ 0 & -1\end{array}\right), \mathbf{K}=\left(\begin{array}{ll}0 & 1 \\ 1 & 0\end{array}\right), \mathbf{L}=\left(\begin{array}{cc}0 & -1 \\ 1 & 0\end{array}\right)$ and $\hat{A}_{\mathrm{I}}, \hat{A}_{\mathrm{J}}, \hat{A}_{\mathrm{K}}$ and $\hat{A}_{\mathrm{L}}$ are four coefficients (scalars) which substitution into equation 9 shows to be given by the set of semi-sums and semidifferences

$$
\begin{aligned}
& \hat{A}_{\mathrm{I}}=\left(\hat{a}_{11}+\hat{a}_{22}\right) / 2 \\
& \hat{A}_{\mathrm{J}}=\left(\hat{a}_{11}-\hat{a}_{22}\right) / 2 \\
& \hat{A}_{\mathrm{K}}=\left(\hat{a}_{21}+\hat{a}_{12}\right) / 2 \\
& \hat{A}_{\mathrm{L}}=\left(\hat{a}_{21}-\hat{a}_{12}\right) / 2
\end{aligned}
$$

The symmetric matrix $\hat{\mathbf{B}}$ can be expanded consistently as

$$
\hat{\mathbf{B}}=\hat{B}_{\mathrm{I}} \mathbf{I}+\hat{B}_{\mathrm{J}} \mathbf{J}+\hat{B}_{\mathrm{K}} \mathbf{K}
$$

and similarly for $\hat{\mathbf{C}}$. The coefficients $\hat{B}_{\mathrm{I}}, \hat{B}_{\mathrm{J}}, \hat{B}_{\mathrm{K}}, \hat{C}_{\mathrm{I}}, \hat{C}_{\mathrm{J}}$ and $\hat{C}_{\mathrm{K}}$ are given by equations corresponding to equations 10,11 and 12. We expand $\hat{\mathbf{e}}=\left(\begin{array}{l}\hat{e}_{\mathrm{i}} \\ \hat{e}_{\mathrm{j}}\end{array}\right)$ as

$$
\hat{\mathbf{e}}=\hat{e}_{\mathrm{i}} \mathbf{i}+\hat{e}_{\mathrm{j}} \mathbf{j},
$$

where $\mathbf{i}=\left(\begin{array}{l}1 \\ 0\end{array}\right), \mathbf{j}=\left(\begin{array}{l}0 \\ 1\end{array}\right)$, and similarly for $\hat{\pi}$.

Transformed transferences of form 4 above can then be expanded as

$$
\hat{A}_{\mathrm{I}}\left(\begin{array}{ccc}
\mathbf{I} & \mathbf{O} & \mathbf{0} \\
\mathbf{O} & -\mathbf{I} & \mathbf{0} \\
\mathbf{o}^{\prime} & \mathbf{0}^{\prime} & 0
\end{array}\right)+\hat{A}_{\mathrm{J}}\left(\begin{array}{ccc}
\mathbf{J} & \mathbf{O} & \mathbf{0} \\
\mathbf{O} & -\mathbf{J} & \mathbf{0} \\
\mathbf{o}^{\prime} & \mathbf{o}^{\prime} & 0
\end{array}\right)+\hat{A}_{\mathrm{K}}\left(\begin{array}{ccc}
\mathbf{K} & \mathbf{O} & \mathbf{0} \\
\mathbf{O} & -\mathbf{K} & \mathbf{0} \\
\mathbf{o}^{\prime} & \mathbf{o}^{\prime} & 0
\end{array}\right)+\hat{A}_{\mathrm{L}}\left(\begin{array}{ccc}
\mathbf{L} & \mathbf{O} & \mathbf{0} \\
\mathbf{O} & \mathbf{L} & \mathbf{0} \\
\mathbf{o}^{\prime} & \mathbf{o}^{\prime} & 0
\end{array}\right) .
$$

That there is no minus sign in front of $\mathbf{L}$ in the middle row of the last matrix is a consequence of the fact that $-\mathbf{L}^{\prime}=\mathbf{L}$. Similarly, for transformed transferences of forms 5 and 6 above one has the consistent expansions

$$
\hat{B}_{\mathrm{I}}\left(\begin{array}{ccc}
\mathbf{O} & \mathbf{I} & \mathbf{0} \\
\mathbf{O} & \mathbf{O} & \mathbf{0} \\
\mathbf{o}^{\prime} & \mathbf{o}^{\prime} & 0
\end{array}\right)+\hat{B}_{\mathrm{J}}\left(\begin{array}{ccc}
\mathbf{O} & \mathbf{J} & \mathbf{o} \\
\mathbf{O} & \mathbf{O} & \mathbf{0} \\
\mathbf{o}^{\prime} & \mathbf{o}^{\prime} & 0
\end{array}\right)+\hat{B}_{\mathrm{K}}\left(\begin{array}{ccc}
\mathbf{O} & \mathbf{K} & \mathbf{o} \\
\mathbf{O} & \mathbf{O} & \mathbf{0} \\
\mathbf{o}^{\prime} & \mathbf{o}^{\prime} & 0
\end{array}\right)
$$

and

$$
\hat{C}_{\mathrm{I}}\left(\begin{array}{ccc}
\mathbf{O} & \mathbf{O} & \mathbf{0} \\
\mathbf{I} & \mathbf{O} & \mathbf{0} \\
\mathbf{o}^{\prime} & \mathbf{o}^{\prime} & 0
\end{array}\right)+\hat{C}_{\mathrm{J}}\left(\begin{array}{ccc}
\mathbf{O} & \mathbf{O} & \mathbf{o} \\
\mathbf{J} & \mathbf{O} & \mathbf{o} \\
\mathbf{o}^{\prime} & \mathbf{o}^{\prime} & 0
\end{array}\right)+\hat{C}_{\mathrm{K}}\left(\begin{array}{ccc}
\mathbf{O} & \mathbf{O} & \mathbf{0} \\
\mathbf{K} & \mathbf{O} & \mathbf{0} \\
\mathbf{o}^{\prime} & \mathbf{o}^{\prime} & 0
\end{array}\right) .
$$

Forms 7 and 8 become

$$
\hat{e}_{\mathrm{i}}\left(\begin{array}{ccc}
\mathbf{O} & \mathbf{O} & \mathbf{i} \\
\mathbf{O} & \mathbf{O} & \mathbf{0} \\
\mathbf{o}^{\prime} & \mathbf{o}^{\prime} & 0
\end{array}\right)+\hat{e}_{\mathrm{j}}\left(\begin{array}{ccc}
\mathbf{O} & \mathbf{O} & \mathbf{j} \\
\mathbf{O} & \mathbf{O} & \mathbf{o} \\
\mathbf{o}^{\prime} & \mathbf{o}^{\prime} & 0
\end{array}\right)
$$

and

$$
\hat{\pi}_{\mathrm{i}}\left(\begin{array}{ccc}
\mathbf{O} & \mathbf{O} & \mathbf{0} \\
\mathbf{O} & \mathbf{O} & \mathbf{i} \\
\mathbf{o}^{\prime} & \mathbf{O}^{\prime} & 0
\end{array}\right)+\hat{\pi}_{\mathrm{j}}\left(\begin{array}{ccc}
\mathbf{O} & \mathbf{O} & \mathbf{o} \\
\mathbf{O} & \mathbf{O} & \mathbf{j} \\
\mathbf{o}^{\prime} & \mathbf{o}^{\prime} & 0
\end{array}\right) .
$$

Any transformed transference $\hat{\mathbf{T}}$ can be expressed as the sum of expressions 14 to 18 . Hence we have the ordered basis for the linear space of transformed transferences: 


$$
\begin{aligned}
& \beta=\left\{\left(\begin{array}{ccc}
\mathbf{I} & \mathbf{O} & \mathbf{0} \\
\mathbf{O} & -\mathbf{I} & \mathbf{0} \\
\mathbf{o}^{\prime} & \mathbf{o}^{\prime} & 0
\end{array}\right),\left(\begin{array}{ccc}
\mathbf{J} & \mathbf{O} & \mathbf{0} \\
\mathbf{O} & -\mathbf{J} & \mathbf{0} \\
\mathbf{o}^{\prime} & \mathbf{o}^{\prime} & 0
\end{array}\right),\left(\begin{array}{ccc}
\mathbf{K} & \mathbf{O} & \mathbf{0} \\
\mathbf{O} & -\mathbf{K} & \mathbf{0} \\
\mathbf{o}^{\prime} & \mathbf{o}^{\prime} & 0
\end{array}\right),\left(\begin{array}{ccc}
\mathbf{L} & \mathbf{O} & \mathbf{0} \\
\mathbf{O} & \mathbf{L} & \mathbf{0} \\
\mathbf{o}^{\prime} & \mathbf{o}^{\prime} & 0
\end{array}\right),\right. \\
& \left(\begin{array}{lll}
\mathbf{O} & \mathbf{I} & \mathbf{0} \\
\mathbf{O} & \mathbf{O} & \mathbf{0} \\
\mathbf{o}^{\prime} & \mathbf{o}^{\prime} & 0
\end{array}\right),\left(\begin{array}{ccc}
\mathbf{O} & \mathbf{J} & \mathbf{0} \\
\mathbf{O} & \mathbf{O} & \mathbf{0} \\
\mathbf{o}^{\prime} & \mathbf{o}^{\prime} & 0
\end{array}\right),\left(\begin{array}{ccc}
\mathbf{O} & \mathbf{K} & \mathbf{0} \\
\mathbf{O} & \mathbf{O} & \mathbf{0} \\
\mathbf{O}^{\prime} & \mathbf{O}^{\prime} & 0
\end{array}\right), \\
& \left(\begin{array}{ccc}
\mathbf{O} & \mathbf{O} & \mathbf{0} \\
\mathbf{I} & \mathbf{O} & \mathbf{0} \\
\mathbf{0}^{\prime} & \mathbf{0}^{\prime} & 0
\end{array}\right),\left(\begin{array}{ccc}
\mathbf{O} & \mathbf{O} & \mathbf{0} \\
\mathbf{J} & \mathbf{O} & \mathbf{0} \\
\mathbf{O}^{\prime} & \mathbf{O}^{\prime} & 0
\end{array}\right),\left(\begin{array}{ccc}
\mathbf{O} & \mathbf{O} & \mathbf{0} \\
\mathbf{K} & \mathbf{O} & \mathbf{0} \\
\mathbf{o}^{\prime} & \mathbf{o}^{\prime} & 0
\end{array}\right), \\
& \left(\begin{array}{ccc}
\mathbf{O} & \mathbf{O} & \mathbf{i} \\
\mathbf{O} & \mathbf{O} & \mathbf{0} \\
\mathbf{O}^{\prime} & \mathbf{0}^{\prime} & 0
\end{array}\right),\left(\begin{array}{lll}
\mathbf{O} & \mathbf{O} & \mathbf{j} \\
\mathbf{O} & \mathbf{O} & \mathbf{0} \\
\mathbf{O}^{\prime} & \mathbf{O}^{\prime} & 0
\end{array}\right) \\
& \left.\left(\begin{array}{lll}
\mathbf{O} & \mathbf{O} & \mathbf{0} \\
\mathbf{O} & \mathbf{O} & \mathbf{i} \\
\mathbf{O}^{\prime} & \mathbf{o}^{\prime} & 0
\end{array}\right),\left(\begin{array}{ccc}
\mathbf{O} & \mathbf{O} & \mathbf{0} \\
\mathbf{O} & \mathbf{O} & \mathbf{j} \\
\mathbf{o}^{\prime} & \mathbf{o}^{\prime} & 0
\end{array}\right)\right\}
\end{aligned}
$$

The set $\beta$ consists of 14 basic transformed transferences. Represented explicitly as $5 \times 5$ matrices $\beta$ is

$$
\begin{aligned}
& \beta=\left\{\begin{array}{lllll}
1 & 0 & 0 & 0 & 0 \\
0 & 1 & 0 & 0 & 0 \\
0 & 0 & -1 & 0 & 0 \\
0 & 0 & 0 & -1 & 0 \\
0 & 0 & 0 & 0 & 0
\end{array}\right),\left(\begin{array}{ccccc}
1 & 0 & 0 & 0 & 0 \\
0 & -1 & 0 & 0 & 0 \\
0 & 0 & -1 & 0 & 0 \\
0 & 0 & 0 & 1 & 0 \\
0 & 0 & 0 & 0 & 0
\end{array}\right),\left(\begin{array}{ccccc}
0 & 1 & 0 & 0 & 0 \\
1 & 0 & 0 & 0 & 0 \\
0 & 0 & 0 & -1 & 0 \\
0 & 0 & -1 & 0 & 0 \\
0 & 0 & 0 & 0 & 0
\end{array}\right),\left(\begin{array}{ccccc}
0 & -1 & 0 & 0 & 0 \\
1 & 0 & 0 & 0 & 0 \\
0 & 0 & 0 & -1 & 0 \\
0 & 0 & 1 & 0 & 0 \\
0 & 0 & 0 & 0 & 0
\end{array}\right), \\
& \left(\begin{array}{lllll}
0 & 0 & 1 & 0 & 0 \\
0 & 0 & 0 & 1 & 0 \\
0 & 0 & 0 & 0 & 0 \\
0 & 0 & 0 & 0 & 0 \\
0 & 0 & 0 & 0 & 0
\end{array}\right),\left(\begin{array}{ccccc}
0 & 0 & 1 & 0 & 0 \\
0 & 0 & 0 & -1 & 0 \\
0 & 0 & 0 & 0 & 0 \\
0 & 0 & 0 & 0 & 0 \\
0 & 0 & 0 & 0 & 0
\end{array}\right),\left(\begin{array}{ccccc}
0 & 0 & 0 & 1 & 0 \\
0 & 0 & 1 & 0 & 0 \\
0 & 0 & 0 & 0 & 0 \\
0 & 0 & 0 & 0 & 0 \\
0 & 0 & 0 & 0 & 0
\end{array}\right), \\
& \left(\begin{array}{lllll}
0 & 0 & 0 & 0 & 0 \\
0 & 0 & 0 & 0 & 0 \\
1 & 0 & 0 & 0 & 0 \\
0 & 1 & 0 & 0 & 0 \\
0 & 0 & 0 & 0 & 0
\end{array}\right),\left(\begin{array}{ccccc}
0 & 0 & 0 & 0 & 0 \\
0 & 0 & 0 & 0 & 0 \\
1 & 0 & 0 & 0 & 0 \\
0 & -1 & 0 & 0 & 0 \\
0 & 0 & 0 & 0 & 0
\end{array}\right),\left(\begin{array}{lllll}
0 & 0 & 0 & 0 & 0 \\
0 & 0 & 0 & 0 & 0 \\
0 & 1 & 0 & 0 & 0 \\
1 & 0 & 0 & 0 & 0 \\
0 & 0 & 0 & 0 & 0
\end{array}\right), \\
& \left(\begin{array}{lllll}
0 & 0 & 0 & 0 & 1 \\
0 & 0 & 0 & 0 & 0 \\
0 & 0 & 0 & 0 & 0 \\
0 & 0 & 0 & 0 & 0 \\
0 & 0 & 0 & 0 & 0
\end{array}\right),\left(\begin{array}{lllll}
0 & 0 & 0 & 0 & 0 \\
0 & 0 & 0 & 0 & 1 \\
0 & 0 & 0 & 0 & 0 \\
0 & 0 & 0 & 0 & 0 \\
0 & 0 & 0 & 0 & 0
\end{array}\right),
\end{aligned}
$$




$$
\left.\left(\begin{array}{lllll}
0 & 0 & 0 & 0 & 0 \\
0 & 0 & 0 & 0 & 0 \\
0 & 0 & 0 & 0 & 1 \\
0 & 0 & 0 & 0 & 0 \\
0 & 0 & 0 & 0 & 0
\end{array}\right),\left(\begin{array}{lllll}
0 & 0 & 0 & 0 & 0 \\
0 & 0 & 0 & 0 & 0 \\
0 & 0 & 0 & 0 & 0 \\
0 & 0 & 0 & 0 & 1 \\
0 & 0 & 0 & 0 & 0
\end{array}\right)\right\} .
$$

We also have a set of 14 coefficients which constitute the 14-entry coordinate vector

$$
\hat{\mathbf{v}}=\left(\begin{array}{llllllllllllll}
\hat{A}_{\mathrm{I}} & \hat{A}_{\mathrm{J}} & \hat{A}_{\mathrm{K}} & \hat{A}_{\mathrm{L}} & \hat{B}_{\mathrm{I}} & \hat{B}_{\mathrm{J}} & \hat{B}_{\mathrm{K}} & \hat{C}_{\mathrm{I}} & \hat{C}_{\mathrm{J}} & \hat{C}_{\mathrm{K}} & \hat{e}_{\mathrm{i}} & \hat{e}_{\mathrm{j}} & \hat{\pi}_{\mathrm{i}} & \left.\hat{\pi}_{\mathrm{j}}\right)^{\prime}
\end{array}\right.
$$

corresponding to basis $\beta$.

Quantitative analysis can be performed for optical systems in the transformed transference space. For example, change of a system or differences between systems can be represented as differences, $\Delta \hat{\mathbf{T}}=\hat{\mathbf{T}}_{2}-\hat{\mathbf{T}}_{1}$ for example. Also, for a set of $N$ systems a mean transformed transference can be obtained from

$$
\widetilde{\hat{\mathbf{T}}}=\frac{1}{N} \sum_{n=1}^{N} \hat{\mathbf{T}}_{n} \text {. }
$$

One can also work with coordinate vectors $\hat{\mathbf{v}}_{n}$ : the first 10 coordinates in equation 20 are given by equations 10 to 13 or their equivalents and the last four are the entries in $\hat{\mathbf{e}}_{n}$ and $\hat{\pi}_{n}$. Then a change or difference is represented as a vector: $\Delta \hat{\mathbf{v}}=\hat{\mathbf{v}}_{2}-\hat{\mathbf{v}}_{1}$. A sample mean can be calculated by

$$
\tilde{\hat{\mathbf{v}}}=\frac{1}{N} \sum_{n=1}^{N} \hat{\mathbf{v}}_{n} .
$$

and a sample variance-covariance by the $14 \times 14$ symmetric matrix

$$
\hat{\mathbf{S}}=\frac{1}{N-1} \sum_{n=1}^{N}\left(\hat{\mathbf{v}}_{n}-\tilde{\hat{\mathbf{v}}}_{n}\right)\left(\hat{\mathbf{v}}_{n}-\tilde{\hat{\mathbf{v}}}_{n}\right)^{\prime} \text {. }
$$

In general $\hat{\mathbf{S}}$ has 14 variances on the diagonal and 91 distinct covariances off the diagonal. Hypotheses on means and variances-covariances can be tested in the transformed transference space.

If the effects of prismatic and decentred elements are of no interest then the basis reduces to the first 10 of the members of $\beta$ in equation 19 and the coordinate vector reduces to the first 10 of the coordinates in equation $20 . \hat{\mathbf{S}}$ reduces to $10 \times 10$ with 10 variances and 45 covariances.

The mathematics suggests a systematic terminology. In equation $9 \hat{A}_{\mathrm{I}} \mathbf{I}$ is a scalar matrix. It is appropriate to call it the scalar component of the submatrix $\hat{\mathbf{A}}$ of the transformed transference $\hat{\mathbf{T}}$ and to call $\hat{A}_{\mathrm{I}}$ the scalar coefficient of $\hat{\mathbf{A}}$. The eigenmeridians of $\hat{A}_{\mathrm{J}} \mathbf{J}$ are 'ortho' (at $0^{\circ}$ and $90^{\circ}$ ) and the eigenvalues are 'anti' $\left(\hat{A}_{\mathrm{J}}\right.$ and $\left.-\hat{A}_{\mathrm{J}}\right)$. Hence it is appropriate to call $\hat{A}_{\mathrm{J}} \mathbf{J}$ the ortho-antistigmatic component and $\hat{A}_{\mathrm{J}}$ the ortho-antistigmatic coefficient of submatrix $\hat{\mathbf{A}}$. With eigenmeridians that are oblique $\left(45^{\circ}\right.$ and $\left.135^{\circ}\right)$ and eigenvalues also 'anti' $\hat{A}_{\mathrm{K}} \mathbf{K}$ is the oblique antistigmatic component of $\hat{\mathbf{A}} . \hat{A}_{\mathrm{K}}$ is the oblique antistigmatic coefficient and $\hat{A}_{\mathrm{L}}$ the antisymmetric coefficient of $\hat{\mathbf{A}}$.

Similar terminology applies in the case of submatrices $\hat{\mathbf{B}}$ and $\hat{\mathbf{C}}$ although the antisymmetric components are null and the antisymmetric coefficients zero. $\hat{e}_{\mathrm{i}}$ and $\hat{e}_{\mathrm{j}}$ are the Cartesian coordinates of submatrix $\hat{\mathbf{e}}$, often the horizontal and vertical coefficients respectively, and similarly for coordinates $\hat{\pi}_{\mathrm{i}}$ and $\hat{\pi}_{\mathrm{j}}$ of submatrix $\hat{\pi}$.

In order to work in the transformed transference space one first transforms the transferences $\mathbf{T}_{n}$ of the optical systems to transformed transferences $\hat{\mathbf{T}}_{n}$ using one of the operations described elsewhere ${ }^{1-3}$. Some 
results, differences and means, for example, can be transformed back to transferences via the inverse operation. It is important that the transformation employed in obtaining any results is stated or clearly understood.

\section{Acknowledgements}

This paper is based on research supported by the National Research Foundation under grant 2053699 to WF Harris. I thank JR Cardoso, A Rubin, RD van Gool and SD Mathebula for continuing discussions.

\section{References}

1. Harris WF. The average eye. Ophthal Physiol Opt 200424 580-585.

2. Harris WF, Cardoso JR. The exponential-mean-log-transference as a possible representation of the optical character of an average eye. Ophthal Physiol Opt 200626 380-383.

3. Cardoso JR, Harris WF. Transformations of ray transferences of optical systems to augmented Hamiltonian matrices and the problem of the average system. S Afr Optom 200766 in 56-61.

4. Mathebula SD, Rubin A, Harris WF. Quantitative analysis in Hamiltonian space of the ray transference of a cornea. S Afr Optom 200766 in $68-76$. 